\title{
Bacterial translocation during enteral and parenteral nutrition
}

\author{
J. Wesley Alexander* \\ Transplantation Division, Department of Surgery, University of Cincinnati College of Medicine, \\ Cincinnati, Ohio 45267-0558, USA
}

\section{Microbial translocation}

Microbial translocation can be defined as the passage of both viable and non-viable microbes and microbial products across the intact intestinal barrier. In human subjects, translocation may be important in the hypermetabolic response to injury, a septic state in the absence of a defined focus, the development of distant infections, and multiple organ failure. The load of microbial products, such as endotoxin, and their role in inciting inflammatory and cytokine responses may be as important or more important than the presence of the organisms themselves.

Translocation is not a new phenomenon; it was vaguely suggested by the writings of Fraenkel (1891). Several more papers in the early 1900 s suggested that organisms could penetrate through the intact bowel wall (Alexander \& Gennari, 1996), but it was really the work of Arnold and his colleagues in 1928-30 (Arnold, 1928, 1930; Arnold \& Brody, 1928) that provided a clear indication for the role of translocation in producing systemic illness in animals. Of interest, he was able to show that alkalinization of the intestinal contents significantly increased the incidence of translocation, and that translocation occurred very early, as soon as 5 min after introduction of bacteria into the lumen of the intestine. Flory (1933) was able to show that translocation occurred through a trans-epithelial process, i.e. through the enterocytes, rather than between them. In the 1950s, Jacob Fine and his colleagues (Fine et al. 1952) established compelling relationships between translocation and irreversible haemorrhagic shock.

Numerous diseases have been found to be associated with translocation in man (Alexander \& Gennari, 1996). These include pneumatosis intestinalis, non-occlusive intestinal gangrene, necrotizing enterocolitis, radiation injury, response to cytotoxic drugs, the cytokine release syndrome, Crohn's disease, ulcerative colitis, haemorrhagic shock, severe trauma, cerebral injury, leukaemia, neutropenia, and colon cancer.

Translocation from the intestine is most commonly detected by measuring the presence of viable bacteria in the tissues. This can reflect not only the integrity of the intrinsic barrier function of the mucosa but also the numbers and types of microbes in the lumen and the ability of the host to kill the bacteria that do translocate. Translocation may occur by three mechanisms: through $\mathrm{M}$ cells, which is a normal processing pathway; through epithelial cells, which increases after systemic injury; through ulcerations, which is an important pathway after cellular injury, such as caused by cytotoxic drugs or radiation (Alexander \& Gennari, 1996). Translocation through the tight junctions has not been observed. Factors that increase microbial translocation, as measured by bacterial counts in the tissues, include: diminished blood flow or $\mathrm{O}_{2}$ delivery, e.g. haemorrhagic shock, burn injury, administration of zymosan or endotoxin, mesenteric occlusion, hypoxia or fever; immunosuppression, e.g. high-dose prednisone or blood transfusions; changes in the intestinal microbial density, e.g. following antibiotic therapy or the administration of an elemental diet or total parenteral nutrition (TPN); direct damage to the epithelium, e.g. irradiation, cytotoxic drugs, irritants, cytomegalovirus infection, mucosal disease, bowel manipulation, bowel obstruction or reperfusion injury (Alexander \& Gennari, 1996).

Drugs which will decrease bacterial translocation can be divided into several broad categories: those which will improve the microcirculation of the intestine, e.g. enalopril or prostaglandin $E_{1}$ or $E_{2}$ analogues; those which increase mucus production, e.g. prostaglandins $E_{1}$ or $E_{2}$ analogues, sucralfate; those which act as growth factors (basic fibrobast growth factor, granulocyte macrophage colony-stimulating factor, epidermal growth factor, bombesin or insulin-like growth factor-1); those which decrease inflammatory damage, e.g. allopurinol, or anti-interleukin 6 . Antibiotics themselves do not seem to influence the mucosal barrier, except via alteration of the numbers and types of microbes in the intestinal lumen (Alexander \& Gennari, 1996).

\section{The process of Candidal translocation}

We have studied the process of the translocation of Candida albicans in the jejunum and ileum of guinea-pigs and rats with thermal injury (Alexander et al. 1990). Suspensions of Candida were introduced into the intestinal lumen either orally or into an isolated Thiry-Vella loop, and the animals were killed at intervals thereafter to study how the Candida crossed the intestinal barrier, using both scanning and transmission electron microscopy as well as light microscopy. Within minutes of introduction, the Candida began to become attached to the epithelial membrane. Through an apparent enzymic process, the Candidal bodies eroded the microvilli and began to become embedded in this layer. Fragments of the microvilli could be seen attached to the outer

Abbreviations: TEN, total enteral nutrition; TPN, total parenteral nutrition.

*Corresponding author: Dr J. Wesley Alexander, fax +1 513558 3580, email Alexanjw@Healthall.com 
membrane of the Candida. Once the Candidal body reached the outer membrane, it was quickly incorporated and passed through the cytoplasm of the enterocytes without morphological injury to the enterocyte itself. They were then extruded into the lamina propria where they were engulfed by macrophages or remained free, passing into the lumens of the central lacteals or small venules. Some of the Candida were incorporated and engulfed by $\mathbf{M}$ cells, whereas others entered through micro-ulcerations at the villus tips. Some formed small micro-abscesses in the submucosal area, whereas others were carried to distant tissues, such as the regional lymph nodes where they were found primarily engulfed within macrophages. They could often be found budding, evidence of invasive growth. Studies with both fluorescent-labelled endotoxin and intact bacteria showed that these translocated in a similar manner, although it was more difficult to study.

\section{Translocation in man}

That translocation occurs in man is without controversy. This is perhaps most clearly demonstrated by a study in which a human volunteer was administered orally approximately $10^{12}$ Candida albicans (Krause et al. 1969). Positive blood cultures for Candida occurred at between 3 and $6 \mathrm{~h}$, and funguria occurred at between 2.75 and $3.25 \mathrm{~h}$. Symptoms lasted for $2-9 \mathrm{~h}$ despite vigorous treatment. Sedman et al. (1994) studied the prevalence of gut translocation in patients before gastrointestinal surgery. Samples of the intestinal mucosa and mesenteric lymph nodes were taken in 257 consecutive surgical patients. Translocation occurred in $10.3 \%$ of the evaluable patients. Post-operative sepsis was significantly more common in those patients with translocation $(28 \%)$ compared with those that had no translocation $(11.5 \%)$.

\section{Early enteral feeding reduces translocation}

Previous studies in our laboratory had shown that early enteral feeding reduced the hypermetabolic response in burned guinea-pigs (Mochizuki et al. 1984). A subsequent study was done to determine whether or not this was related to translocation from the intestinal tract (Gianotti et al. 1994). Guinea-pigs were provided with a gastrostomy, and 2 weeks later a $40 \%$ total body surface area thermal burn was inflicted. They were then divided into two groups. One received a complete enteral diet by gastrostomy, whereas the other received an equal volume of Ringer's lactate solution. Both treatments started immediately post-burn and continued for $48 \mathrm{~h}$. Escherichia coli $\left(10^{10}\right.$, labelled with $\left.{ }^{14} \mathrm{C}\right)$ were then administered through the gastrostomy tube. Resting metabolic expenditure was determined $4 \mathrm{~h}$ after administration of $E$. coli. Radioactivity in the portal blood and ileal mucosa and also plasma cortisol levels, urinary vanalyl mandelic acid and intestinal mucosa weight were determined $5 \mathrm{~h}$ after administration of $E$. coli. The mucosal weight was significantly lower in those animals receiving Ringer's lactate solution when compared with those receiving enteral nutrition. There was a strong inverse correlation between the amount of radioactive material within the mucosa and the mucosal weight for individual animals, indicating that as the mucosa atrophied, translocation increased. There was also a strong inverse correlation between mucosal weight and the amount of radioactivity in the portal blood. Not surprisingly, the amount of radioactivity in the mucosa and blood correlated significantly, and in starved animals these tissues had greater amounts of radioactivity than in the fed animals. The amount of radioactivity in the blood and the mucosa correlated significantly with the urinary vanalyl mandelic acid and cortisol, and radioactivity in the blood and tissues also directly correlated with the resting energy expenditure. These studies gave a clear indication that translocation of enteric organisms was an important trigger for the hypermetabolic response following burn injury.

These animal studies are further supported by the findings of a clinical study by Chiarelli (1990). These investigators compared early enteral nutrition with delayed enteral nutrition in burn patients. Twenty patients with burn injuries averaging $38 \%$ were divided into two groups. The early-fed group received nutrition starting an average of $4.4($ SE 0.5$) \mathrm{h}$ post-burn, whereas the delayed-fed group received enteral nutrition beginning 57.7 (SE 2.6) h post-burn. There were five positive blood cultures from three patients in the earlyfed group compared with thirty-three from seven patients of the delayed-fed group. Early feeding decreased the duration of hospitalization from 89 to $69 \mathrm{~d}$. As in the animal studies, glucagon was normalized in the patients fed early, while it remained elevated in patients for whom feeding was delayed. Early enteral feeding also has been found to be beneficial in a variety of other conditions, such as head injury. Grahm et al. (1989) did a randomized study in patients with head injuries in which the experimental group was fed with a naso-jejunal tube within $36 \mathrm{~h}$ and the control group was fed by gastric tube when bowel sounds began spontaneously. Infection occurred in fourteen patients in the control group compared with only three patients in the experimental group, and the period spent in the Intensive Care Unit decreased from 10 to $7 \mathrm{~d}$. Studies by Inoue et al. (1989) showed that a single bolus of food given either 3 or $12 \mathrm{~h}$ post-burn could decrease the incidence of translocation from the intestine. Another study in burned guinea-pigs (Inoue et al. 1991) showed that early enteral feeding would reduce the incidence of both translocation and mortality in burned guinea-pigs that were administered Candida albicans orally. Thus, in injured subjects, early enteral nutrition will decrease translocation, improve survival, and decrease the hypermetabolic response which occurs as a consequence of translocation.

\section{Total parenteral nutrition $v$. total enteral nutrition and the role of translocation}

In a large multicentre study, Busby (1991) evaluated the role of TPN $v$. no TPN in patients before surgery. TPN was given pre- and post-operatively for $10 \mathrm{~d}$. There was no difference in the total complication rate in the two groups of patients, but the $90 \mathrm{~d}$ mortality rate was slightly higher in the TPN group $(13.4 \% v .10 .5 \%)$ and infectious complications were significantly higher in the TPN group (14.1\% v. $6.4 \%$; $P=0 \cdot 01$ ). Sandstrom et al. (1993) randomized 300 patients to receive either TPN or intravenous glucose post-operatively for up to $15 \mathrm{~d}$. There was no difference in outcome when analysed by intent to treat, but the patients 
who received only glucose for $15 \mathrm{~d}$ had a higher mortality. These studies indicated that in many surgical patients, TPN not only lacked beneficial effect, but might have harmful effects. Alverdy et al. (1988) suggested that one of the harmful effects from TPN might be that it promotes bacterial translocation from the gut. In an animal study, he showed that spontaneous translocation from the intestine exceeded $60 \%$ in animals that received TPN. If the same animals were fed the TPN solution enterally, the incidence of translocation was reduced by half. Control animals receiving regular chow did not show evidence of translocation. This is consistent with numerous subsequent studies which showed that intravenous nutrition increases translocation and also that elemental-type diets are associated with increased translocation, although not as much as diets given by the total parenteral route. Pappo et al. (1994) fed rats for $7 \mathrm{~d}$ before oral administration of $1.5 \times 10^{10}$ Candida. Animals receiving a control enteral diet had a low incidence of translocation to the kidneys, but animals receiving TPN with or without antibiotics had significantly higher rates of translocation to blood, lymph nodes and kidneys.

Comparisons of the clinical benefit of total enteral nutrition (TEN) $v$. TPN have been done by several investigators. One of the first of these was by Moore et al. (1989). They randomized trauma patients to receive either TEN or TPN beginning $12 \mathrm{~h}$ post-injury. The patients receiving TEN had only a $9 \%$ incidence of infections overall compared with a $37 \%$ incidence in the patients with TPN. Perhaps the most extensive study was that of Kudsk et al. (1992), also in seriously injured patients. His patients had both a central line and a feeding jejunostomy tube placed at the time of laparotomy for blunt or penetrating abdominal trauma. Patients were then divided randomly to receive either TEN or TPN. The incidence of pneumonia, line sepsis, and intra-abdominal abscess were all significantly less in those receiving TEN, and the benefit was greatest in patients with larger injuries.

These studies are all consistent with the concept that TPN increases translocation compared with TEN, and its associated with a greater incidence of septic complications.

\section{Selected enteral nutrients decrease translocation and improve survival}

\section{Arginine}

The effect of arginine on survival rates and host defence mechanisms was studied using two clinically-relevant models of infection that included transfusion-induced immunosuppression (Gianotti et al. 1993). Balb/c mice were fed for $10 \mathrm{~d}$ on a defined AIN-76A diet, an AIN-76A diet supplemented with arginine ( $2 \%$ dietary energy), an AIN-76A diet supplemented with glycine ( $4 \%$ dietary energy), or standard laboratory chow. In most experiments, the mice were then transfused with allogeneic blood and allowed to feed for an additional $5 \mathrm{~d}$ before undergoing either caecal ligation and puncture or oral administration of $10^{10} \mathrm{E}$. coli and a $20 \%$ burn injury. Additional animals receiving the argininesupplemented diet were treated with the NO inhibitor $\mathrm{N} \omega$-nitro-L-arginine before oral administration of $E$. coli and burn injury. The effect of these diets and $\mathrm{N} \omega$-nitro-L-arginine on the degree of translocation of ${ }^{14} \mathrm{C}$-radiolabelled $E$. coli from the intestine and the ability of the host to kill translocated organisms was also investigated. Mice were fed and received the transfusion, oral administration of $E$. coli, and burn injury as described previously. Mesenteric lymph nodes, liver and spleen were harvested $4 \mathrm{~h}$ post-burn.

Survival after caecal ligation and puncture was $56 \%$ in the arginine-supplemented group $v .28 \%$ in the AIN-76A group and $20 \%$ in the chow group $(P<0.02)$. After oral administration of $E$. coli and burn injury, survival was $100 \%$ in the arginine-supplemented group v. $50 \%$ in both the glycinesupplemented and chow-fed groups and $35 \%$ in the AIN$76 \mathrm{~A}$-fed group $(P<0.01)$. In animals receiving the argininesupplemented diet, treatment with $\mathrm{N} \omega$-nitro-L-arginine decreased survival from $95 \%$ to $30.5 \%(P<0.0001)$. Greater translocation, as measured by radioactivity, was observed to the mesenteric lymph nodes of the AIN-76A-fed group. However, there was no difference in translocation to the liver and spleen related to dietary group. Quantitative colony counts and the calculated percentage of remaining viable bacteria showed that the ability to kill translocated organisms was significantly enhanced in animals receiving arginine.

\section{Glutamine}

Similar studies were done with glutamine using regular rat chow, an AIN-76A diet and an AIN-76A diet supplemented with glycine or glutamine ( $2 \%$ dietary energy; Gianotti et al. 1995). Animals were fed for 2 weeks before a $20 \%$ burn injury and oral administration of ${ }^{14} \mathrm{C}$-labelled bacteria. A transfusion was given to provide additional mild immunosuppression. As in the experiments with arginine, the intestine was the only source of bacteria to cause infection. All the animals prefed with glutamine survived compared with only $20-40 \%$ of the animals in the control groups. When the spleen, liver and mesenteric lymph nodes were removed and measured for radioactivity, it was found that the animals receiving glutamine had significantly less translocation to these tissues, as well as fewer colony counts, indicating that this amino acid significantly reduced translocation from the intestine and also improved the killing of bacteria.

Another experiment was done in which prednisone $(10 \mathrm{mg} / \mathrm{kg}$ ) was given for $4 \mathrm{~d}$ before challenge by oral administration of $10^{9} \mathrm{E}$. coli and a $20 \%$ burn injury (Gennari \& Alexander, 1997). Controls received diets enriched with glycine. Survival for the groups were as follows: arginine $64 \%$ v. glycine $24 \%(P=0.004)$; glutamine $55 \% v$. glycine $20 \%(P=0.015)$. These studies indicated that single dietary amino acids could improve survival even in immunosuppressed animals.

\section{n-3 Fatty acids}

The effect of dietary lipids on bacterial translocation, killing of translocated organisms and host survival was studied in a burned animal model (Gianotti et al. 1996). Balb/c mice were fed on one of the three experimental AIN-76A diets (containing $15 \%$ dietary energy from fish oil, safflower oil or a 50:50 mixture i.e. each oil provided $7.5 \%$ dietary energy), AIN-76A without added lipids, or a non-purified 
Table 1. Effects of selected nutrients on translocation of bacteria

\begin{tabular}{lccc}
\hline & $\begin{array}{c}\text { Effect on } \\
\text { barrier function }\end{array}$ & $\begin{array}{c}\text { Effect on } \\
\text { bacterial killing }\end{array}$ & $\begin{array}{c}\text { Effect on } \\
\text { survival }\end{array}$ \\
\hline Arginine & - & $\uparrow$ & $\uparrow$ \\
Glutamine & $\uparrow$ & $\uparrow$ & $\uparrow$ \\
RNA* & - & - & - \\
$n$-3 Fatty acids & $\uparrow$ & $\uparrow$ & $\uparrow$ \\
$n$-6 Fatty acids & - & - & - \\
Glycine & - & $N D$ & - \\
Dietary fibre (insoluble) & $N D$ & $\uparrow$ & $\uparrow$ \\
Arginine + glutamine & $\uparrow$ & $\uparrow$ & $\uparrow$ \\
Fish oil + arginine & $\uparrow$ & $\uparrow$ & $\uparrow$ \\
Fish oil + glutamine & $\uparrow$ &
\end{tabular}

ND, not determined; $\uparrow$, effect was increased; - , no effect.

* Considered a nutrient in that it occurs in natural foodstuffs and provides synthetic building blocks.

stock diet. All animals were transfused on day 10. On day 15, the animals were orally administered $10^{10} \mathrm{E}$. coli labelled with ${ }^{14} \mathrm{C}$ and given a $20 \%$ burn injury. Survival was $84 \%$ in the fish oil-fed group $v .36 \%$ in the safflower oil- and $50: 50$ mixture-fed groups, and $25 \%$ and $20 \%$ in the two control groups $(P<0.0001)$. The numbers of viable translocating bacteria were reduced in all tested organs in the fish oil-fed groups compared with the other groups.

\section{Combinations of dietary additives}

Experiments were done to determine whether combinations of individual nutrients would have an additive effect in improving survival from translocation in a burned animal model (Gennari et al. 1995). This model used a $30 \%$ burn injury rather than a $20 \%$ burn injury. Diets containing arginine plus fish oil or fish oil plus glutamine were associated with significantly higher survival than control diets. Interestingly, when medium-chain triacylglycerols were added to the diets with either arginine or glycine, no beneficial effect was obtained. The effects of various enteral nutrients on translocation are summarized in Table 1.

\section{Acknowledgements}

This work was supported in part by USPHS Grant AI-12936.

\section{References}

Alexander JW, Boyce ST, Babcock GF, Gianotti L, Peck MD, Dunn DL, Pyles T, Childress CP \& Ash SK (1990) The process of microbial translocation. Annals of Surgery 212, 496-512.

Alexander JW \& Gennari R (1996) Translocation as it applies to metabolism. In Nutrition and Metabolism in the Surgical Patient, 2nd ed., pp. 459-476 [JE Fischer, editor]. Boston, MA: Little, Brown and Company.

Alverdy JC, Aoys E \& Moss GS (1988) Total parenteral nutrition promotes bacterial translocation from the gut. Surgery 104, $185-190$

Arnold L (1928) The passage of living bacteria through the wall of the intestine and the influence of diet and climate upon intestinal auto-disinfection. American Journal of Hygiene 8, 605-632.
Arnold L (1930) Alterations in the endogenous enteric bacterial flora and microbic permeability of the intestinal wall in relation to the nutritional and meteorological changes. Journal of Hygiene 29, 82-116.

Arnold L \& Brody L (1928) Passage of bacteria through the intestinal mucosa. Proceedings of the Society for Experimental Biology and Medicine 24, 832.

Busby G (1991) Perioperative total parenteral nutrition in surgical patients. The Veterans' Affairs Total Parenteral Nutrition Cooperative Study Group. New England Journal of Medicine 325, 525-532.

Chiarelli AP (1990) Very early enteral nutrition supplementation in burned patients. American Journal of Clinical Nutrition 51 , 1035-1039.

Fine J, Frank H, Schweinburg F, Jacob S \& Gordon T (1952) The bacterial factor in traumatic shock. Annals of the New York Academy of Sciences 55, 429-445.

Flory HW (1933) Observations on the functions of mucus and the early stages of bacterial invasion of the intestinal mucosa. Journal of Pathology and Bacteriology 37, 283-289.

Fraenkel A (1891) Ueber peritoneale infektion (On peritoneal infection). Weiner Klinische Wochenschrift 4, 241-265.

Gennari R \& Alexander JW (1997) Arginine, glutamine, and DHEA reverse the immunosuppressive effect of prednisone during gut derived sepsis. Critical Care Medicine 25, 1207-1214.

Gennari R, Alexander JW \& Eaves-Pyles T (1995) Effect of different combinations of dietary additives on bacterial translocation and survival in gut-derived sepsis. Journal of Parenteral and Enteral Nutrition 19, 319-325.

Gianotti L, Alexander JW, Gennari R, Pyles T \& Babcock GF (1995) Oral glutamine decreases bacterial translocation and improves survival in experimental gut-origin sepsis. Journal of Parenteral and Enteral Nutrition 14, 69-74.

Gianotti L, Alexander JW, Pyles T \& Fukushima R (1993) Arginine-supplemented diets improve survival in gut-derived sepsis and peritonitis by modulating bacterial clearance: the role of nitric oxide. Annals of Surgery 217, 644-654.

Gianotti L, Alexander JW, Pyles T \& Fukushima R (1996) Dietary fatty acids modulate host bactericidal response, microbial translocation and survival following blood transfusion and thermal injury. Clinical Nutrition 15, 291-296.

Gianotti L, Nelson JL, Alexander JW, Chalk CL \& Pyles T (1994) Post injury hypermetabolic response and magnitude of translocation: prevention by early enteral nutrition. Nutrition 3 , 225-231.

Grahm TW, Zadrozny DB \& Harrington T (1989) The benefits of early jejunal hyperalimentation in the head injured patient. Neurosurgery $25,729-735$.

Inoue S, Epstein MD, Alexander JW, Trocki O, Jacobs P \& Gura P (1989) Prevention of yeast translocation across the gut by a single enteral feeding after burn injury. Journal of Parenteral and Enteral Nutrition 13, 565-571.

Inoue S, Peck M \& Alexander JW (1991) Fungal translocation is associated with increased mortality after thermal injury in guinea pigs. Journal of Burn Care and Rehabilitation 12, 19-22.

Krause W, Matheis H \& Wulf K (1969) Fungaemia and funguria after oral administration of Candida albicans. Lancet $\mathbf{i}, 598-599$.

Kudsk KA, Croce MA, Fabian TC, Minard G, Tolley EA, Poret HA, Kuhl MR \& Brown RO (1992) Enteral vs. parenteral feeding. Effects on septic morbidity after blunt and penetrating abdominal trauma. Annals of Surgery 215, 503--511.

Mochizuki H, Trocki O, Dominioni L, Brackett KA, Joffe SN \& Alexander JW (1984) Mechanisms of prevention of postburn hypermetabolism and catabolism by early enteral feeding. Annals of Surgery 200, 297-310. 
Moore FA, Moore EE, Jones TN, McCroskey BL \& Peterson VM (1989) TEN versus TPN following major abdominal trauma reduced septic morbidity. Journal of Trauma 29, 916-922.

Pappo I, Polacheck I, Zmora O, Feigin E \& Freund HR (1994) Altered gut barrier function to Candida during parenteral nutrition. Nutrition 10, 151-154.

Sandstrom R, Drott C, Hyltander A, Arfvidsson B, Schersten T,
Wickstrom I \& Lundholm K (1993) The effect of postoperative intravenous feeding (TPN) on outcome following major surgery evaluated in a randomized study. Annals of Surgery 217, 185-195.

Sedman PC, Macfie J, Sagar P, Mitchell CJ, May J, Mancey-Jones B \& Johnstone D (1994) The prevalence of gut translocation in humans. Gastroenterology 107, 643-649.

() Nutrition Society 1998 